\title{
Competitive and Cooperative Systems: a mini-review
}

\author{
Morris W. Hirsch ${ }^{1}$ and Hal L. Smith ${ }^{2}$ \\ 1 Department of Mathematics, University of California, Berkeley, CA, 94720, \\ hirsch@math. berkeley .edu \\ 2 Department of Mathematics, Arizona State University, Tempe, AZ 85287, USA \\ Institute 2, halsmith@asu.edu
}

\begin{abstract}
The theory of competitive and cooperative dynamical systems has had some remarkable applications to the biological sciences. The interested reader may consult the monograph [28] and lecture notes [29] of Smith, and to a forthcoming review by the authors [11] for a more in-depth treatment.
\end{abstract}

\section{Strong Monotonicity for ODEs}

In this brief review we give some of the main results in the theory of competitive and cooperative systems. But first, we give some new strong monotonicity results for odes. Let $J$ be a nontrivial open interval, $D \subset \mathbb{R}^{\mathrm{n}}$ be an open set, $f: J \times D \rightarrow \mathbb{R}^{\mathrm{n}}$ be a locally Lipschitz function, and consider the ordinary differential equation

$$
x^{\prime}=f(t, x)
$$

Denote by $x\left(t, t_{0}, x_{0}\right)$ the non-continuable solution of the initial value problem $x\left(t_{0}\right)=x_{0}$ for $t_{0} \in J$.

A cone $K$ in $\mathbb{R}^{\mathrm{n}}$ is a non-empty, closed subset of $\mathbb{R}^{\mathrm{n}}$ satisfying $K+K \subset K$, $\mathbb{R} \cdot \mathrm{K} \subset \mathrm{K}$ and $K \cap(-K)=\{0\}$. We hereafter assume $K$ nonempty interior in $\mathbb{R}^{\mathrm{n}}$. The order relations $\leq,<, \ll$ are induced by $K$ as follows: $x \leq y$ if and only if $y-x \in K ; x<y$ if $x \leq y$ and $x \neq y$, and $x \ll y$ whenever $y-x \in \operatorname{Int} K$. A cone is a polyhedral cone if it is the intersection of a finite family of half spaces. The standard cone $\mathbb{R}_{+}^{\mathrm{n}}=\cap_{\mathrm{i}=1}^{\mathrm{n}}\left\{\mathrm{x}:\left\langle\mathrm{e}_{\mathrm{i}}, \mathrm{x}\right\rangle \geq 0\right\}$ is polyhedral $\left(e_{i}\right.$ is the unit vector in the $x_{i}$-direction) while the ice cream cone $K=\left\{x \in \mathbb{R}^{\mathrm{n}}\right.$ : $\left.\mathrm{x}_{1}^{2}+\mathrm{x}_{2}^{2}+\cdots+\mathrm{x}_{\mathrm{n}-1}^{2} \leq \mathrm{x}_{\mathrm{n}}^{2}, \mathrm{x}_{\mathrm{n}} \geq 0\right\}$ is not. The dual cone $K^{*}$ is the set positive linear functionals, i.e., linear functionals $\lambda \in\left(\mathbb{R}^{\mathrm{n}}\right)^{*}$, the dual space of $\mathbb{R}^{\mathrm{n}}$, such that $\lambda(K) \geq 0$. If we adopt the standard inner product $\langle$,$\rangle on \mathbb{R}^{\mathrm{n}}$ then we can identify $\left(\mathbb{R}^{\mathrm{n}}\right)^{*}$ with $\mathbb{R}^{\mathrm{n}}$ since for each $\lambda \in K^{*}$ we can find $a \in \mathbb{R}^{\mathrm{n}}$ such that $\lambda(x)=\langle a, x\rangle$ for all $x$. We use the following easy result; see e.g. Walcher [35]. 
Lemma 1. Let $x \in K$. Then $x \in$ IntK if and only if $\lambda(x)>0$ for all $\lambda \in$ $K^{*} \backslash\{0\}$.

We say that (1) is monotone, or order-preserving, if whenever $x_{0}, x_{1} \in D$ satisfy $x_{0} \leq x_{1}$ and the solutions $x\left(t, t_{0}, x_{0}\right)$ and $x\left(t, t_{0}, x_{1}\right)$ are defined on $\left[t_{0}, t_{1}\right], t_{1}>t_{0}$, then $x\left(t, t_{0}, x_{0}\right) \leq x\left(t, t_{0}, x_{1}\right)$ holds for $t \in\left[t_{0}, t_{1}\right]$.

The vector field $f: J \times D \rightarrow \mathbb{R}^{\mathrm{n}}$ is said to satisfy the quasimonotone condition in $D$ if for every $(t, x),(t, y) \in J \times D$ we have

(Q) $x \leq y$ and $\phi(x)=\phi(y)$ for some $\phi \in K^{*}$ implies $\phi(f(t, x)) \leq \phi(f(t, y))$.

The quasimonotone condition was introduced by Schneider and Vidyasagar [25] for finite dimensional, autonomous linear systems and used later by Volkmann [34] for nonlinear infinite dimensional systems. The following result is certainly inspired by a result of Volkmann [34] and work of W. Walter [?]. See also Uhl [33] and Walcher [35]. The proof appears in [11].

Theorem 1.1 Let $f$ satisfies $(Q)$ in $D, t_{0} \in J$, and $x_{0}, x_{1} \in D$. Let $<_{r}$ denote any one of the relations $\leq,<, \ll$. If $x_{0}<_{r} x_{1}$ and $t>t_{0}$ are such that both $x\left(t, t_{0}, x_{i}\right), i=0,1$ are defined, then $x\left(t, t_{0}, x_{0}\right)<_{r} x\left(t, t_{0}, x_{1}\right)$. Conversely, if (1) is monotone, then $f$ satisfies $(Q)$.

It will be useful to specialize to linear systems since it is convenient in the applications to verify $(\mathrm{Q})$, as well as other monotonicity hypotheses, using derivatives. Let $A(t)$ be a continuous matrix-valued function defined on the interval $J$ containing $t_{0}$ and consider the linear initial value problem for the matrix solution $X$ :

$$
X^{\prime}=A(t) X, \quad X\left(t_{0}\right)=I .
$$

For linear systems, $(\mathrm{Q})$ is equivalent to the following:

(M)for all $x \in \partial K$ and $\lambda \in K^{*}$ such that $\lambda(x)=0, \lambda(A(t) x) \geq 0$.

Therefore, we have the following Corollary of Theorem 1.4.

Corollary 1.1 The matrix solution $X(t)$ satisfies $X(t) K \subset K$ for $t \geq t_{0}$ for each $t_{0} \in J$ if and only if for all $t \in J,(M)$ holds for the function $x \rightarrow A(t) x$. In fact, $(M)$ implies that $X(t)(K \backslash\{0\}) \subset(K \backslash\{0\})$ and $X(t) \operatorname{Int} K \subset \operatorname{IntK}$ for $t>t_{0}$.

A matrix $A$ is $K$-nonnegative if $A(K) \subset K$. Corollary 1.1 says that $X(t)$ is $K$-nonnegative for $t \geq t_{0}$ if (M) holds.

The domain $D$ is $p$-convex if for every $x, y \in D$ satisfying $x \leq y$ the line segment joining them also belongs to $D$. Let $\frac{\partial f}{\partial x}(t, x)$ be continuous on $J \times D$. We say that $f$ (or (1)) is $K$-cooperative if for all $t \in J, y \in D$, (M) holds for the function $x \rightarrow \frac{\partial f}{\partial x}(t, y) x$. By Corollary 1.1 applied to the variational equation

$$
X^{\prime}(t)=\frac{\partial f}{\partial x}\left(t, x\left(t, t_{0}, x_{0}\right)\right) X, \quad X\left(t_{0}\right)=I
$$

we conclude that if $f$ is $K$-cooperative then $X(t)=\frac{\partial x}{\partial x_{0}}\left(t, t_{0}, x_{0}\right)$ is $K$-positive. Straightforward arguments lead to the following result. 
Theorem 1.2 Let $\frac{\partial f}{\partial x}(t, x)$ be continuous on $J \times D$. Then $(Q)$ implies that $f$ is $K$-cooperative. Conversely, if $D$ is p-convex and $f$ is $K$-cooperative, then (Q) holds.

If $K=\mathbb{R}_{+}^{\mathrm{n}}$, then it is easy to see by using the standard inner product that we may identify $K^{*}$ with $K$. The quasimonotone hypothesis reduces to the Kamke condition [22,14]: $x \leq y$ and $x_{i}=y_{i}$ implies that $f_{i}(t, x) \leq$ $f_{i}(t, y)$. This holds by taking $\phi(x)=\left\langle e_{i}, x\right\rangle$ and noting that every $\phi \in K^{*}$ can be represented as a positive linear combination of these functionals. If $f$ is differentiable, the Kamke condition implies

$$
\frac{\partial f_{i}}{\partial x_{j}}(t, x) \geq 0, i \neq j
$$

Conversely, if $\frac{\partial f}{\partial x}(t, x)$ is continuous on $J \times D$ and satisfies (3) and if $D$ is p-convex, then the Kamke condition holds.

Stern and Wolkowicz [32] give necessary and sufficient conditions for (M) to hold for matrix $A$ relative to the ice cream cone $K=\left\{x \in \mathbb{R}^{\mathrm{n}}: \mathrm{x}_{1}^{2}+\right.$ $\left.\mathrm{x}_{2}^{2}+\cdots+\mathrm{x}_{\mathrm{n}-1}^{2} \leq \mathrm{x}_{\mathrm{n}}^{2}, \mathrm{x}_{\mathrm{n}} \geq 0\right\}$. Let $Q$ denote the $n \times n$ diagonal matrix with first $n-1$ entries 1 and last entry -1 . Then (M) holds for $A$ if and only if $Q A+A^{T} Q+\alpha Q$ is negative semidefinite for some $\alpha \in \mathbb{R}$. Their characterization extends to other ellipsoidal cones.

Additional hypotheses are required for establishing the strong order preserving property and here we provide full details following [11]. Recall that the matrix $A$ is strongly positive if $A(K \backslash\{0\}) \subset \operatorname{Int} K$. The following hypothesis for the matrix $A$ follows Schneider and Vidyasagar [25].

(T)for all $x \neq 0, x \in \partial K$, there exists $\nu \in K^{*}$ such that $\nu(x)=0$ and $\nu(A x)>0$.

Our next result was proved by Elsner [3] for the case of constant matrices, answering a question in [25]. Our proof follows that of Theorem 4.3.26 of Berman et al [1].

Proposition 1.1 Let the linear system (2) satisfy (M). Then the fundamental matrix $X\left(t_{1}\right)$ is strongly positive for $t_{1}>t_{0}$ if there exists $s$ satisfying $t_{0}<$ $s \leq t_{1}$ such that $(T)$ holds for $A(s)$.

Proof: If not, there exists $x>0$ such that the solution of (2) given by $y(t)=$ $X(t) x$ satisfies $y\left(t_{1}\right) \in \partial K \backslash\{0\}$. By Corollary 1.1, $y(t)>0$ for $t \geq t_{0}$ and $y(t) \in \partial K$ for $t_{0} \leq t \leq t_{1}$. Let $s \in\left(t_{0}, t_{1}\right]$ be such that $(\mathrm{T})$ holds for $A(s)$. Then there exists $\nu \in K^{*}$ such that $\nu(y(s))=0$ and $\nu(A(s) y(s))>0$. As $\nu \in K^{*}$ and $y(t) \in K, h(t):=\nu(y(t)) \geq 0$ for $t_{0} \leq t \leq t_{1}$. But $h(s)=0$ and $\left.\frac{d}{d t}\right|_{t=s} h(t)=\nu(A(s) y(s))>0$ which, taken together, imply that $h(s-\delta)<0$ for small positive $\delta$, giving the desired contradiction.

Proposition 1.2 leads immediately to a result on strong monotonicity for the nonlinear system (1). 
Theorem 1.3 Let $D$ be $p$-convex, $\frac{\partial f}{\partial x}(t, x)$ be continuous on $J \times D$, and $f$ be $K$-cooperative. Let $B=\left\{(t, x) \in J \times D:(T)\right.$ does not hold for $\left.\frac{\partial f}{\partial x}(t, x)\right\}$. Suppose that for all $\left(t_{0}, x_{0}\right) \in J \times D$, the set $\left\{t>t_{0}:\left(t, x\left(t, t_{0}, x_{0}\right)\right) \in B\right\}$ is nowhere dense. Then $x\left(t, t_{0}, x_{0}\right) \ll x\left(t, t_{0}, x_{1}\right)$ for $t>t_{0}$ for which both solutions are defined provided $x_{0}, x_{1} \in D$ satisfy $x_{0}<x_{1}$. In particular, this holds if $B$ is empty.

Proof: We apply the formula

$$
x\left(t, t_{0}, x_{1}\right)-x\left(t, t_{0}, x_{0}\right)=\int_{0}^{1} \frac{\partial x}{\partial x_{0}}\left(t, t_{0}, s x_{1}+(1-s) x_{0}\right)\left(x_{1}-x_{0}\right) d s
$$

where $X(t)=\frac{\partial x}{\partial x_{0}}\left(t, t_{0}, y_{0}\right)$ is the fundamental matrix for (2) corresponding to the matrix $A(t)=\frac{\partial f}{\partial x}\left(t, x\left(t, t_{0}, y_{0}\right)\right)$. The left hand side belongs to $K \backslash\{0\}$ if $x_{0}<x_{1}$ by Theorem 1.5 and Theorem 1.4 but we must show it belongs to Int $K$. For this to be true, it suffices that for each $t>t_{0}$ there exists $s \in[0,1]$ such that the matrix derivative in the integrand is strongly positive. In fact, it is $K$-positive by Corollary 1.1 for all values of the arguments with $t \geq t_{0}$ so application of any nontrivial $\phi \in K^{*}$ to the integral gives a nonnegative numerical result. If the condition mentioned above holds then the application of $\phi$ to the integrand gives a positive numerical result for all $s^{\prime}$ near $s$ by continuity and Lemma 1.1 and hence the integral belongs to Int $K$ by Lemma 1.1. By Proposition 1.2, $\frac{\partial x}{\partial x_{0}}\left(t, t_{0}, y_{0}\right)$ is strongly positive for $t>t_{0}$ if $(\mathrm{T})$ holds for $A(r)=\frac{\partial f}{\partial x}\left(r, x\left(r, t_{0}, y_{0}\right)\right)$ for some $r \in\left(t_{0}, t\right]$. But this is guaranteed by our hypotheses.

The somewhat stronger condition of irreducibility may be more useful in applications because there is a large body of theory related to it $[2,1]$. A closed subset $F$ of $K$ that is itself a cone is called a face of $K$ if $x \in F$ and $0 \leq y \leq x$ (inequalities induced by $K$ ) implies that $y \in F$. For example, the faces of $K=\mathbb{R}_{+}^{\mathrm{n}}$ are of the form $\left\{x \in \mathbb{R}_{+}^{\mathrm{n}}: \mathrm{x}_{\mathrm{i}}=0, \mathrm{i} \in \mathrm{I}\right\}$ where $I \subset\{1,2, \cdots n\}$. For the ice-cream cone $K=\left\{x \in \mathbb{R}^{\mathrm{n}}: \mathrm{x}_{1}^{2}+\mathrm{x}_{2}^{2}+\cdots+\mathrm{x}_{\mathrm{n}-1}^{2} \leq\right.$ $\left.\mathrm{x}_{\mathrm{n}}^{2}, \mathrm{x}_{\mathrm{n}} \geq 0\right\}$, the faces are the rays issuing from the origin and passing through its boundary vectors. A $K$-positive matrix $A$ is $K$-irreducible if the only faces $F$ of $K$ for which $A(F) \subset F$ are $\{0\}$ and $K$. The famous Perron-Frobenius Theory is developed for $K$-positive and $K$-irreducible matrices in Berman and Plemmons [2]. In particular, the spectral radius of $A$ is a simple eigenvalue of $A$ with corresponding eigenvector in $\operatorname{Int} K$. The next result is adapted from Theorem 4.3.17 of Berman et al. [1].

Proposition 1.2 Let $A$ be a matrix such that $B:=A+\alpha I$ is $K$-positive for some $\alpha \in \mathbb{R}$. Then $B$ is $K$-irreducible if and only if $(T)$ holds for $A$.

Motivated by Proposition 1.3, we introduce the following hypothesis for matrix $A$. 
(I) There exists $\alpha \in \mathbb{R}$ such that $A+\alpha I$ is $K$-positive and $K$-irreducible.

In the special case that $K=\mathbb{R}_{+}^{\mathrm{n}}, n \geq 2$, matrix $A$ satisfies (I) if and only if $a_{i j} \geq 0$ for $i \neq j$ and for every non-empty, proper subset $I$ of $N:=\{1,2, \cdots, n\}$, there is an $i \in I$ and $j \in N \backslash I$ such that $a_{i j} \neq 0$. This is equivalent to the assertion that the incidence graph of $A$ is strongly connected. See Berman and Plemmons [2].

The following is a direct corollary of Theorem 1.6.

Corollary 1.2 Let $D$ be p-convex, $\frac{\partial f}{\partial x}(t, x)$ be continuous on $J \times D$ and $f$ be $K$-cooperative. Let $\tilde{B}=\left\{(t, x) \in J \times D:\right.$ (I) does not hold for $\left.\frac{\partial f}{\partial x}(t, x)\right\}$. Suppose that for all $\left(t_{0}, x_{0}\right) \in J \times D$, the set $\left\{t>t_{0}:\left(t, x\left(t, t_{0}, x_{0}\right)\right) \in \tilde{B}\right\}$ is nowhere dense. Then $x\left(t, t_{0}, x_{0}\right) \ll x\left(t, t_{0}, x_{0}\right)$ for $t>t_{0}$ for which both solutions are defined provided $x_{0}, x_{1} \in D$ satisfy $x_{0}<x_{1}$. In particular, this holds if $\tilde{B}$ is empty.

Corollary 1.2 is an improvement of the restriction of Theorem 10 of Kunze and Siegel [15] to the case that $K$ has nonempty interior. Their results also treat the case that $K$ has empty interior in $\mathbb{R}^{\mathrm{n}}$ but nonempty interior in some subspace of $\mathbb{R}^{\mathrm{n}}$.

Theorem 4.3.40 of Berman et al. [1] implies that for polyhedral cones a matrix $A$ satisfies (M) and (T) if and only if there exists $\alpha \in \mathbb{R}$ such that $A+\alpha I$ is $K$-positive and $K$-irreducible. Therefore, for polyhedral cones like $\mathbb{R}_{+}^{\mathrm{n}}$, Corollary 1.2 and Theorem 1.6 are equivalent.

\section{Competitive and Cooperative Systems}

We now focus on the autonomous system of ordinary differential equations

$$
x^{\prime}=f(x)
$$

where $f$ is continuously differentiable on an open subset $D \subset \mathbb{R}^{\mathrm{n}}$. Let $\phi_{t}(x)$ denote the solution of (4) that starts at the point $x$ at $t=0$. $\phi_{t}$ will be referred to as the flow corresponding to (4). We sometimes refer to $f$ as the vector field generating the flow $\phi_{t}$. We introduce following mild dissipativity condition for our next result.

(A)For each $x \in D, \phi_{t}(x)$ is defined for all $t \geq 0$ and $\phi_{t}(x) \in D$. Moreover, for each bounded subset $A$ of $D$, there exists a compact subset $B=B(A)$ of $D$ such that for each $x \in A, \phi_{t}(x) \in B$ for all large $t$.

The following result should be viewed as prototypical of the generic convergence result that may be proved using general results in [30, 28].

Theorem 2.1 Let the hypotheses of Theorem 1.6 hold for (4), assume $D=$ $\mathbb{R}^{\mathrm{n}}$ or $D=\mathbb{R}_{+}^{\mathrm{n}}$, and assume that $(A)$ holds. Then the set $C$ of convergent points contains an open and dense subset of $D$. 
We say that (4) is $K$-competitive in $D$ if the time-reversed system $x^{\prime}=$ $-f(x)$ is $K$-cooperative. Observe that if (4) is a $K$-competitive system with flow $\phi_{t}$ then the time reversed system above is a $K$-cooperative system with flow $\psi_{t}$ where $\psi_{t}(x)=\phi_{-t}(x)$, and conversely. Therefore, by time reversal, a competitive system becomes a cooperative system and vice-versa. We will sometimes drop the $K$ from $K$-cooperative (competitive) when no confusion may result.

Let $A$ be an invariant set for (4) with flow $\phi_{t}$ (i.e. $\phi_{t}(A)=A$ for all $t$ ) and let $B$ be an invariant set for the system $y^{\prime}=F(y)$ with flow $\psi_{t}$. We say that the flow $\phi_{t}$ on $A$ is topologically equivalent to the flow $\psi_{t}$ on $B$ if there is a homeomorphism $Q: A \rightarrow B$ such that $Q\left(\phi_{t}(x)\right)=\psi_{t}(Q(x))$ for all $x \in A$ and all $t \in \mathbb{R}$. The relationship of topological equivalence says, roughly, that the qualitative dynamics of the two flows are the same. With these definitions, we can state a result of Hirsch [4].

Theorem 2.2 The flow on a compact limit set of a competitive or cooperative system in $\mathbb{R}^{\mathrm{n}}$ is topologically equivalent to a flow on a compact invariant set of a Lipschitz system of differential equations in $\mathbb{R}^{\mathrm{n}-1}$.

The Poincaré-Bendixson Theorem for three dimensional cooperative and competitive systems is the most notable consequence of Theorem 1.9. It was proved by Hirsch [10] who improved earlier partial results $[4,26]$.

Theorem 2.3 (Poincaré-Bendixson Theorem for 3-Dimensional Competitive and Cooperative Systems) A compact limit set of a competitive or cooperative system in $\mathbb{R}^{3}$ that contains no equilibrium points is a periodic orbit.

The following result of Smith [28] is useful for verifying that an omega limit set is a periodic orbit.

Theorem 2.4 Suppose that $D \subset \mathbb{R}^{3}$ contains a unique equilibrium $p$ for the competitive system (4) and it is hyperbolic. Suppose further that its stable manifold $W^{s}(p)$ is one-dimensional and tangent at $p$ to a vector $v \gg 0$. If the orbit of $q \in D \backslash W^{s}(p)$ has compact closure in $D$, then $\omega(q)$ is a nontrivial periodic orbit.

The existence of $v \gg 0$ usually follows from the Perron-Frobenius Theorem. Zhu and Smith [37] establish the existence of an orbitally asymptotically stable periodic orbit if (4) is dissipative and $f$ is analytic. Ortega and Sánchez [?] observed that the above results hold for general cones.

Competitive systems arise naturally from models in the biological sciences, not just in population biology. The following, taken from de Leenheer and Smith [12], illustrates this point. Consider an individual infected with a virus $V$ which attacks target cells $T$ producing infected cells $T^{*}$ which in turn each produce on average $N$ virus particles during their lifetimes. Following Perelson et al. [24], who focus on HIV, we obtain the following system for the dynamics of the vector of blood-concentrations $\left(T, T^{*}, V\right) \in \mathbb{R}_{+}^{3}$. 


$$
\begin{aligned}
\dot{T} & =f(T)-k V T \\
\dot{T}^{*} & =-\beta T^{*}+k V T \\
\dot{V} & =-\gamma V+N \beta T^{*}-k V T .
\end{aligned}
$$

Perelson et al. [24] take $f(T) \equiv \delta-\alpha T+p T\left(1-\frac{T}{T_{\max }}\right)$ with $\delta, \alpha, p, T_{\max }$ positive and denote by $\bar{T}$ the positive root of $f(T)=0$. The basic reproductive number for the model, $R_{0}=k \bar{T}(N-1) / \gamma$, gives the number of infected $\mathrm{T}$ cells produced by a single infected $\mathrm{T}$ cell in a healthy individual.

Among other results, de Leenheer and Smith [12] prove the following.

Theorem 2.5 If $R_{0}>1$, in addition to the unstable virus-free state $E_{0} \equiv$ $(\bar{T}, 0,0)$, there is a "chronic disease" steady state $E_{e} \equiv\left(T_{e}, T_{e}^{*}, V_{e}\right)$ given by

$$
T_{e}=\bar{T} / R_{0}, \quad T_{e}^{*}=\gamma V_{e} /(N-1) \beta, \quad V_{e}=f\left(T_{e}\right) / k T_{e} .
$$

which is locally attracting if $f^{\prime}\left(T_{e}\right) \leq 0$. The omega limit set of every solution with initial conditions satisfying $T^{*}(0)+V(0)>0$ either contains $E_{e}$ or is a nontrivial periodic orbit. There exist parameter values for which $E_{e}$ is unstable with a two dimensional unstable manifold. In this case, there exists an orbitally asymptotically stable periodic orbit; every solution except those with initial data on the one-dimensional stable manifold of $E_{e}$ or on the $T$ axis converges to a non-trivial periodic orbit.

System (5) is competitive with respect to the cone $K:=\left\{T, V \geq 0, T^{*} \leq\right.$ $0\}$. The change of variables $T^{*} \rightarrow-T^{*}$ results in a system the Jacobian for which has non-positive off-diagonal terms on the relevant domain and hence is competitive in the $\mathbb{R}_{+}^{3}$-sense. In [12], it is shown that $E_{e}$ is unstable with a twodimensional unstable manifold when $k T_{\max }>\beta+\gamma+\frac{2 \gamma}{N-1}$ and $p$ is sufficiently large. The final assertion of Theorem 1.12 follows from Theorem 1.11; domain $D$ is chosen to exclude $E_{0}$. The existence of an orbitally asymptotically stable periodic orbit uses the analyticity of the system and results of [37].

\section{References}

1. A. Berman, M. Neumann and R. Stern, Nonnegative matrices in dynamic systems, John Wiley\& Sons, New York (1989).

2. A. Berman and R. Plemmons, Nonnegative matrices in the mathematical sciences, Academic Press, New York (1979)

3. L. Elsner, Quasimonotonie und Ungleichungen in halbgeordneten Raumen, Linear Alg. Appl. 8(1974), 249-261.

4. M.W. Hirsch, Systems of differential equations which are competitive or cooperative 1: limit sets. SIAM J. Appl. Math. 13(1982), 167-179.

5. M.W. Hirsch, Systems of differential equations which are competitive or cooperative II: convergence almost everywhere. SIAM J. Math. Anal. 16(1985), 423-439. 
6. M.W. Hirsch, Systems of differential equations that are competitive or cooperative. IV: Structural stability in three dimensional systems. SIAM J.Math. Anal.21(1990), 1225-1234.

7. M.W. Hirsch and H.L. Smith, Monotone dynamical systems, in preparation.

8. P. de Leenheer and H.L. Smith, Virus Dynamics: a global analysis, to appear, SIAM J. Appl. Math (2003).

9. R. Loewy and H. Schneider, Positive operators on the $n$-dimensional ice cream cone, J. Math. Anal. \& Appl. 49(1975), 375-392.

10. E. Kamke, Zur Theorie der Systeme Gewoknlicher Differentialgliechungen, II, Acta Math. 58(1932), 57-85.

11. H. Kunze and D. Siegel, Monotonicity with respect to closed convex cones II, Applicable Analysis 77(2001), 233-248.

12. R. Lemmert and P. Volkmann, On the positivity of semigroups of operators, Comment. Math. Univ. Carolinae 39(1998), 483-489.

13. M. Muller, Uber das fundamenthaltheorem in der theorie der gewohnlichen differentialgleichungen, Math. Zeit. 26(1926), 619-645.

14. R. Ortega and L. Sánchez, Abstract competitive systems and orbital stability in $\mathbb{R}^{3}$, Proc. Amer. Math. Soc. 128(2000), 2911-2919.

15. A.S. Perelson and P.W. Nelson, Mathematical analysis of HIV-1 dynamics in vivo, SIAM Rev. 41(1999), 3-44.

16. H. Schneider and M. Vidyasagar, Cross-positive matrices, SIAM J. Numer. Anal. 7(1970), 508-519.

17. H.L. Smith, Periodic orbits of competitive and cooperative systems, J.Diff.Eqns. 65(1986), 361-373.

18. H.L. Smith, Monotone Dynamical Systems, an introduction to the theory of competitive and cooperative systems, Math. Surveys and Monographs, 41, American Mathematical Society, Providence, Rhode Island(1995).

19. H.L. Smith, Dynamics of Competition, in Mathematics Inspired by Biology, Springer Lecture Notes in Math. 1714, 1999, 191-240.

20. H.L. Smith and H.R. Thieme, Convergence for strongly ordered preserving semiflows, SIAM J. Math. Anal. 22(1991), 1081-1101.

21. R. Stern and H. Wolkowicz, Exponential nonnegativity on the ice cream cone, SIAM J. Matrix Anal. Appl. 12(1991), 160-165.

22. R. Uhl, Ordinary differential inequalities and quasimonotonicity in ordered topological vector spaces, Proc. Amer. Math. Soc. 126(1998), 1999-2003.

23. P. Volkmann, Gewohnliche Differentialungleichungen mit quasimonoton wachsenden Funktionen in topologischen Vektorraumen, Math. Z. 127(1972), 157164.

24. S. Walcher, On cooperative systems with respect to arbitrary orderings, Journal of Mathematical Analysis and Appl. 263(2001), 543-554.

25. W. Walter, Differential and Integral Inequalities, Springer-Verlag, Berlin(1970).

26. H.-R. Zhu and H.L. Smith, Stable periodic orbits for a class of three dimensional competitive systems, J.Diff.Eqn. 110(1994), 143-156. 